
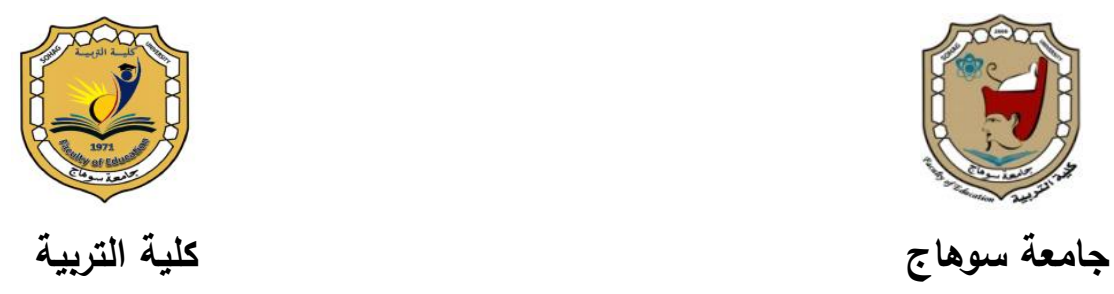

كلية التربية

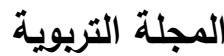

اضطراب الألعاب عبر الإنترنت

\title{
Internet Gaming Disorder (IGD)
}

$$
\text { "إدهان الألعاب الإلكترونية" }
$$

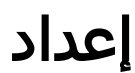

$$
\begin{aligned}
& \text { أ.د / سامية "محمد صابر" محمد عبل النبي } \\
& \text { أستاذ الصحة النفسية } \\
& \text { كلية التربية - جامعة بنها } \\
& \text { جمهورية مصر العربية }
\end{aligned}
$$

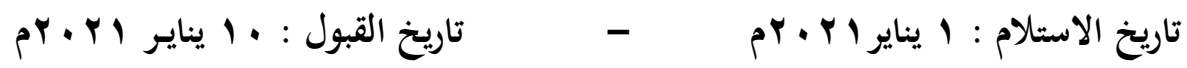

DOI: 10.12816/EDUSOHAG.2021. 
الألعاب عبر الإنترنت أو ألعاب الفيديو هي صناعة عالمية تبلغ قيمتها مليار دولار وهي مستمرة في التوسع والابتكار. (King,\&Delfabbro,2020)

ومع التطور السريع للهواتف الذكية والثبكات اللاسلكية، أصبحت ممارسة الألعاب عبر الإنترنت في أي وقت وفي أي مكان أحد الأثكال الرئيسة للترفيه لاي الأطفال والمراهقين

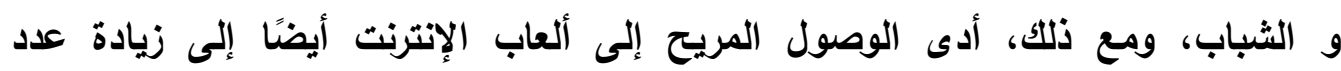

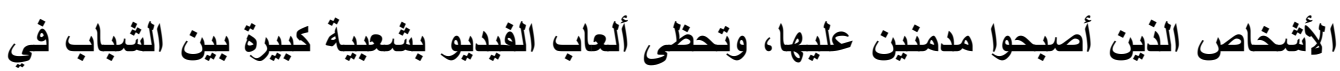

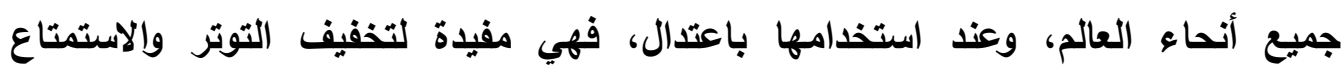
والتواصل مع الآخرين، وتصبح ألعاب الإنترنت مَرضية عند استخدامها بشكل مُفرط، وقد ينتج

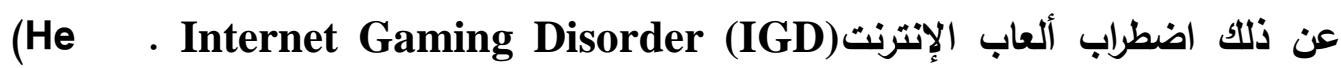
,Pan, Nie, Zheng, \& Chen, 2021)

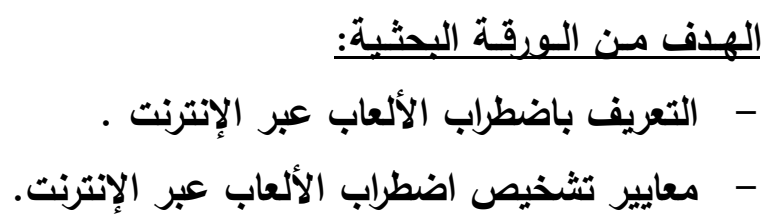

- - تناول بعض من نتائج الدراسات السابقة التي تتاولت اضطراب الألعاب عبر الإنترنت.

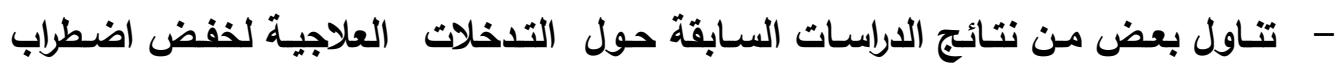

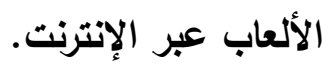
المقترحات والتوصيات: على ضوءالإطار النظري ونتائج الدراسات السابقة تم تقديم عدة مقترحات وتوصيات. 


\title{
abstract \\ Internet gaming disorder \\ Internet Gaming Disorder (IGD) \\ "Addiction to electronic games"
}

\author{
Prepared \\ Samia "Muhammad Saber" Muhammad Abdul Nabi \\ Professor of mental health \\ College of Education - Benha University \\ The Egyptian Arabic Republic
}

\section{Introduction:}

Online gaming or video games is a billion dollar global industry that is constantly expanding and innovating.( King, \& Delfabbro, 2020)

With the rapid development of smartphones and wireless networks, playing online games anytime and anywhere has become one of the main forms of entertainment for children, teenagers and young adults. However, comfortable access to internet games has also led to an increase in the number of people who have become addicted to them. Video is very popular among young people all over the world, and when used in moderation, it is useful for relieving stress, enjoyment and communicating with others, and Internet games become satisfying when used in excess, and this may result in Internet Gaming Disorder (IGD). (He, Pan, Nie, Zheng, \& Chen, 2021)

The goal of the research paper:

Introducing Internet gaming disorder.-

Criteria for diagnosing Internet gaming disorder.-Examine some of the results of previous studies on Internet gaming disorder. -Examine some of the results of previous studies on therapeutic interventions to reduce Internet gaming disorder.

Suggestions and recommendations:

In light of the previous studies and the theoretical framework, several proposals and recommendations were presented. 
الألعاب عبر الإنترنت أو ألعاب الفيديو هي صناعة عالمية تبلغ قيمتها مليار دولار وهي مستمرة في التوسع والابتكار. (King,\&Delfabbro,2020) ومع التطور السريع للهواتف الأكية والثبكات اللاسلكية، أصبحت ممارسة الألعاب عبر الإنترنت في أي وقت وفي أي مكان أحد الأثنكال الرئيسة للترفيه لاي الأطفال والمراهقين

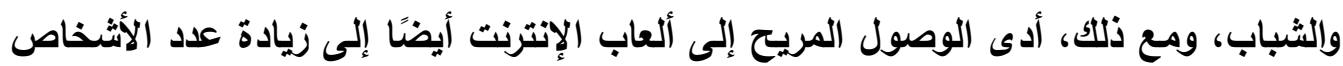
الذين أصبحوا مدمنين عليها، وتحظى ألعاب الفيديو بشعبية كبيرة بين الثباب في جميع

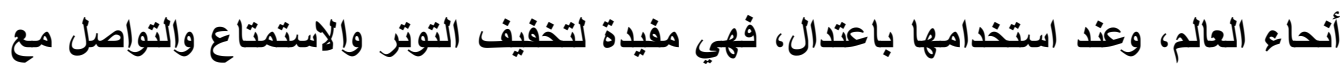

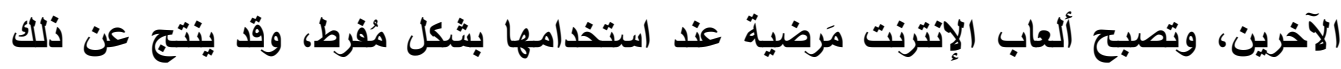

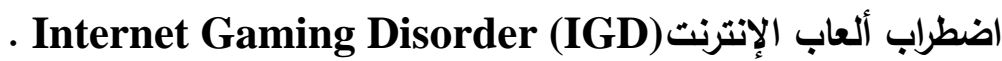
قد أدرجت الجمعية الأمريكية للطب النفسي (APA, 2013) "اضطراب الألعاب عبر الإنترنت" (IGD) في الإصدار

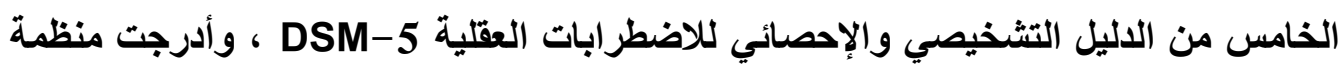

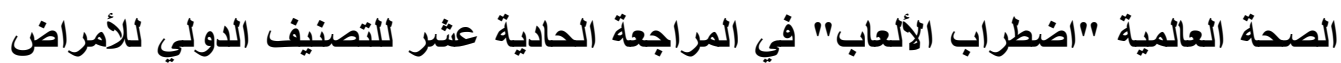

(Zajac, Ginley \& Chang,2020).ICD-11

(World Health Organization, ومنذ أن عَرَفت منظمة الصحة العالمية

2018)

Game Addiction

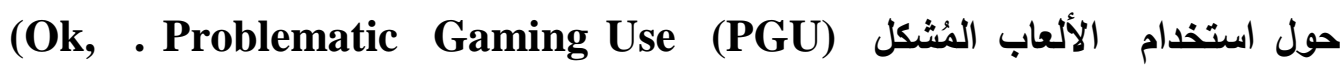
2021)

DSM- في IGD ولا يزال هناك العديد من الخلافات وإلمخاوف المتعلقة بإدراج اضطراب 5، و ينص الاليل 5 - DSM- نفسه على أن الاضطراب الجديد يتطلب مزيدًا من الدراسة،

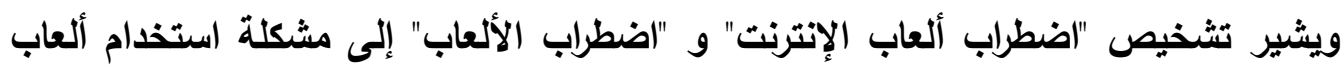
الكمبيوتر، وفي DSM-5، تم سرد تسعة معايير لاضطراب الألعاب عبر الإنترنت وهي:

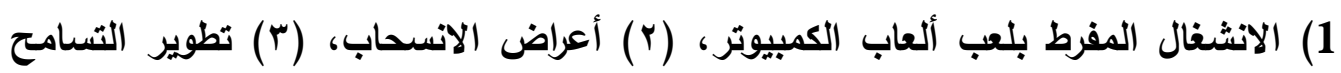

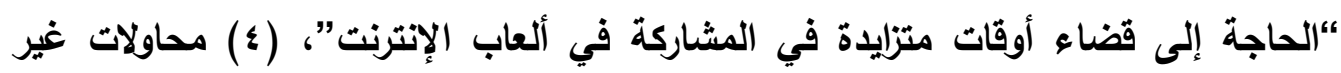
ناجحة للتحم في اللعب، (ه) فقدان الاهتمام بالهوايات والأنشطة الترفيهية (الأخرى) السابقة، (؟) استمرار اللعب المُفرط، على الرغم من التبصر بالعواقب النفسية والاجتماعية، 
خداع الآخرين فيما يتعلق بنطاق اللعب، (^) استخدام ألعاب الكمبيوتر للهروب أو (V)

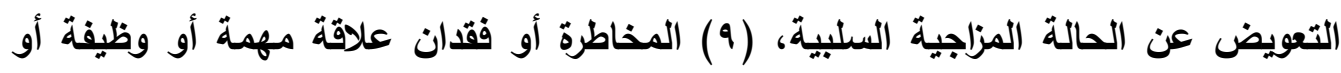
تدريب / فرصة مهنية بسبب اللعب، وإذا تم استيفاء خمسة أو أكثر من هذه المعايير التسعة،

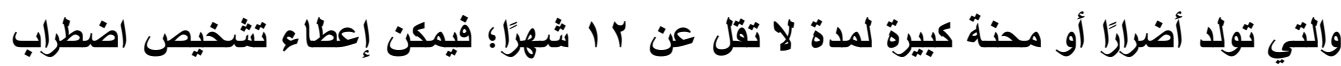

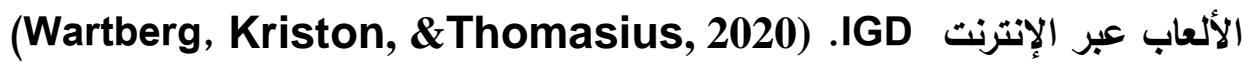
ويرتبط اضطراب الألعاب عبر الإنترنت، بالإحباط اليومي للاحتياجات الأساسية (أي

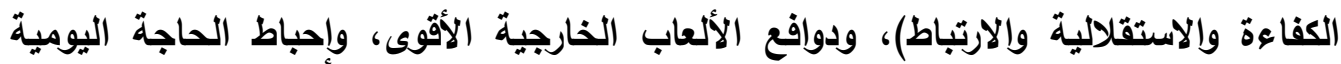
يُقوض ويُضعف ضبط النفس؛ مما يُسهم في ارتفاع اضطراب الألعاب عبر الإنترنت IGD. (Mills, \& Allen, 2020)

ويشير يو وآخرون(Yu,Mo,Zhang,Li,\& Lau, 2021) وميلز وألين (2020 , ) Mills, \& Allen من الأضرار الجسدية والنفسية، ويسبب انخفاض الأداء الأكاديمي والرفاهية الاجتماعية والصحة النفسية والعقلية لاي المراهقين. (Wong,Mo,Potenza, Chan, Lau, وأظهرت نتائج دراسة وونغ وآخرون Chui, Pakpour, \&Lin, 2020)

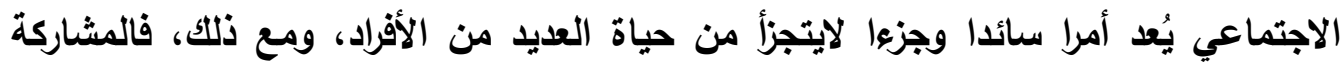

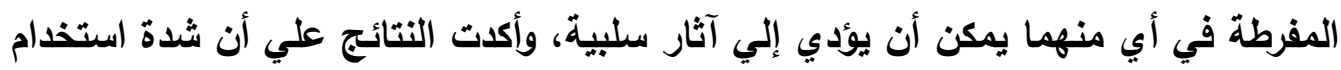

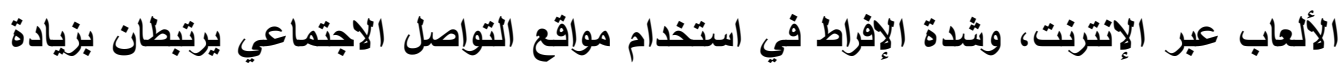
الضغوط النفسية (قلق،اكتئاب،توتر)، وضعف جودة النوم. وأثنارت نتائج دراسة يوان وآخرون (Yuan, Elhai, \& Hall, 2021) إلى أن شدة

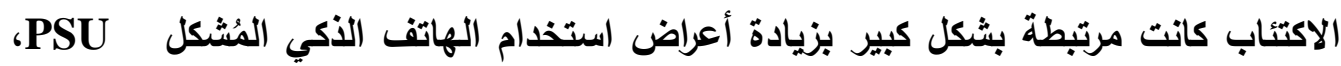
واضطراب الألعاب عبر الإنترنت IGD. كما أوضح وانغ وتثينغ (Wang, \& Cheng, 2021 ) أن كل من القلق الاجتماعي والوحدة النفسية والاكتئاب يرتبط باضطراب الألعاب عبر الإنترنت.

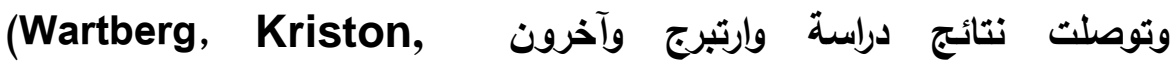
\&Thomasius, 2020) 
وانخفاض وظيفة ودور الأسرة كانت مرتبطة إحصائيًا بشكل كبير مع كل من اضطراب الألعاب عبر الإنترنت IGD واستخدام وسائل التواصل الاجتماعي المُشكل PSMU، وارتبط جنس الأكور وانخفاض التحصيل الدراسي باضطراب الألعاب عبر الإنترنت IGD، وارتبط جنس الأكور باضطراب الألعاب عبر الإنترنت IGD، واستخدام وسائل التواصل الاجتماعي المُشكل PSMU، في حين أن انخفاض العمر، والأعراض الأكثر اكتئابًا، وإنخفاض أداء الأسرة، واضطراب الألعاب عبر الإنترنت IGD كانت مرتبطة باستخدام وسائل التواصل الاجتماعي

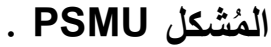

وأسفرت نتائج دراسة كيم وآخرون (Kim,Choi,\&Kim, 2020) عن أن الاستخدام المفرط للهواتف الأكية يرتبط بشكل سلبي بتقدير الذات بين البالغين الذين يعانون من اضطراب ألعاب الإنترنت، وكان الذكور لديهم تقدير ذات منخفض وإفراط في استخدام الهاتف لإنف الأكي أكثر من الإناث، ويُناءً على النتائج، يجب بذل المزيد من الجهود لتقليل الاستخدام المُفرط أو المُشكل للهواتف الذكية من خلال تطوير تدخلات إرشادية وعلاجية، لأولئك الذين الأني يعانون من اضطراب ألعاب الإنترنت. ويشير كنج ودلفابرو ( King,\&Delfabbro, 2020 ) إلي تطور التذخلات الإرشادية والعلاجية الخاصة باضطراب الألعاب عبر الإنترنت بشكل مطرد خلال العقد

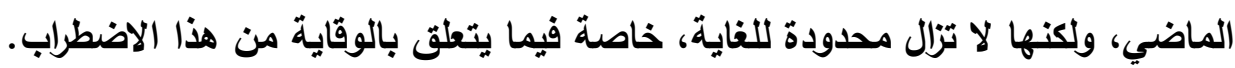

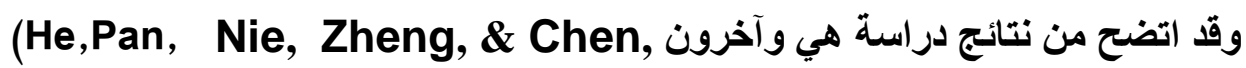
(2021 فعالية التعديل السلوكي، حيث إنه قد انخفضت درجات شدة اضطراب ألعاب الإنترنت ومستوى القلق والثغف لاي الثباب مدمني الألعاب عبر الإنترنت. (Han, Seo,Hawang, Kim, Han, وتبين من خلال نتائج دراسة هان وآخرون الابني (2020 أن العلاج المعرفي السلوكي (CBT) كان علاجًا فعالً لاضطراب ألعاب الإنترنت

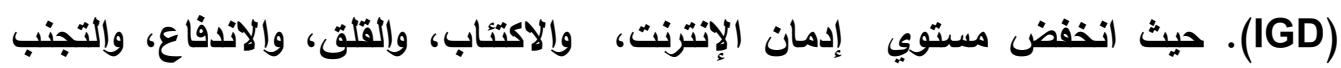
الاجتماعي، وحدث تحسن في مستويات الانتباه والتماسك الأسري. 


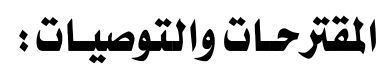

على ضوء الإطار النظري ونتائج الاراسات السابقة بمكن تقديم المقترحات التالية: 1. هناك حاجة إلى مزيد من الاراسات حول اضطراب الألعاب عبر الإنترنت وعلاقته

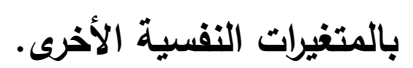
r. ب. دراسة العوامل الكامنة وراء أعراض اضطراب الألعاب عبر الإنترنت.

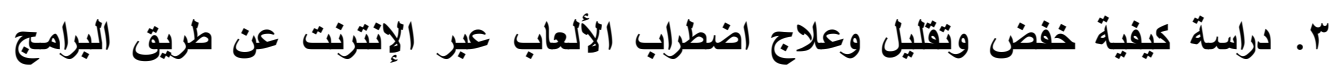
الوقائية والإرشادية والعلاجية. ؛. . دراسة مقارنة بين فعالية أنواع مختلفة من العلاجات النفسية في علاج اضطراب الألعاب

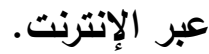




\section{المراجع}

- American Psychiatric Association (APA), (2013). American Psychiatric Association (APA)Diagnostic and statistical manual of mental disorders (DSM-5) (5th ed.), American Psychiatric Association, Washington, DC (2013)

- Han,J.,Seo,Y., Hawang, H., Kim, S.M., \&Han, D.H. (2020). Efficacy of cognitive behavioural therapy for internet gaming disorder.Clinical Psychology \& Psychotherapy, 27, Issue2, March/April 2020, Pages 203213, https://doi.org/10.1002/cpp.2419

- He,J. Pan,T., Nie,Y., Zheng,Y.,\& Chen,S.(2021). Behavioral modification decreases approach bias in young adults with internet gaming disorder. Addictive Behaviors, 113, February 2021, 106686, https://doi.org/10.1016/ j.addbeh. 2020.106686

- Kim,H.,Choi,Y.,\&Kim,D. -J. (2020). Excessive Smartphone Use and SelfEsteem Among Adults With Internet Gaming Disorder: Quantitative Survey Study. JMIR Mhealth Uhealth 2020;8(9):e18505

- King,D.L.\&Delfabbro,P.H.(2020). Chapter 7 - Gaming disorder in young people. (Pages 159-187).In Hodes,M.,Gau,S.S.-F.\&de Vries,P.J. Starting at the Beginning: Laying the Foundation for Lifelong Mental Health. Copyright (c) 2020 Elsevier Inc. All rights reserved. https://doi.org/10.1016/B978-0-12-819749-3.00007-5

- Mills,D.,J.,\& Allen,J.,J. ( $r \cdot r \cdot)$. Self-determination theory, internet gaming disorder, and the mediating role of self-control. Computers in Human Behavior, 105, April 2020, 106209, https://doi.org/ 10.1016/ j.chb.2019.106209

- Ok,C.(2021). Extraversion, loneliness, and problematic game use: A longitudinal study. Personality and Individual Differences, 168, 1 January 2021, 110290 , https://doi.org/10.1016/j.paid.2020.110290

- Wang,H.-Y.,\&Cheng,C.(2021). New perspectives on the prevalence and associated factors of gaming disorder in Hong Kong community adults: A generational approach. Computers in Human Behavior, 114, January 2021, 106574, https:// doi.org/ 10.1016/j.chb.2020.106574

- Wartberg,L., Kriston,L., \&Thomasius,R. (2020). Internet gaming disorder and problematic social media use in a representative sample of German adolescents: Prevalence estimates, comorbid depressive symptoms and related psychosocial aspects. Computers in Human Behavior, 103, February 2020, Pages 31-36, https://doi.org/ 10.1016/j .chb. 2019.09.014

- Wong,H.,Y.,Mo,H.,Y., $\quad$ Potenza,M.,N.,Chan,M.,N.,M.,Lau,W., M.,Chui,T., K., Pakpour, A., H., \& Lin, C.-Y. (2020). Relationships 
between Severity of Internet Gaming Disorder, Severity of Problematic Social Media Use, Sleep Quality and Psychological Distress. Int. J. Environ. Res. Public Health 2020, 17(6), 1879; https://doi.org/10.3390/ijerph17061879

- Yu,Y., Mo, P.K.H., Zhang,J., Li,J.\&Lau, J.T.F.(2021). Why is Internet gaming disorder more prevalent among Chinese male than female adolescents? The role of cognitive mediators. Addictive Behaviors,112, January 2021, 106637, https://doi.org/10.1016/j .addbeh .2020 .106637

- Yuan, G., Elhai, J.D., \& Hall, B., J. (2021). The influence of depressive symptoms and fear of missing out on severity of problematic smartphone use and Internet gaming disorder among Chinese young adults: A threewave mediation model. Addictive Behaviors, Volume 112, January 2021, 106648, https://doi.org/ 10.1016/j.addbeh. 2020.106648.

- Zajac,K., Ginley,M.,K., \& Chang,R. (2020). Treatments of internet gaming disorder: a systematic review of the evidence. Journal Expert Review of Neurotherapeutics,20,2020-Issue 1 